\title{
Positron detection for the intraoperative localisation of cancer deposits
}

\author{
Morand Piert • Maria Burian • \\ Günther Meisetschläger • Hubert J. Stein • \\ Sibylle Ziegler • Jörg Nährig • Maria Picchio • \\ Andreas Buck • Joerg R. Siewert • Markus Schwaiger
}

Received: 25 November 2006 / Accepted: 22 February 2007 / Published online: 12 April 2007

(C) Springer-Verlag 2007

\begin{abstract}
Purpose The study investigated the feasibility of a positron-sensitive hand-held detector system for the intraoperative localisation of tumour deposits resulting from intravenous $\left[{ }^{18} \mathrm{~F}\right] \mathrm{FDG}$ administration.

Methods A total of 17 patients (12 receiving preoperative $\left[{ }^{18} \mathrm{~F}\right]$ FDG PET imaging) with various histologically proven malignancies were included. Radioactivity from tumours and surrounding normal tissue was measured on average $3 \mathrm{~h}$ after administration of $36-110 \mathrm{MBq}\left[{ }^{18} \mathrm{~F}\right] \mathrm{FDG}$ and the tumour-to-background (T/B) ratio was calculated. In addi-
\end{abstract}

M. Piert $(\bowtie) \cdot$ G. Meisetschläger $\cdot$ S. Ziegler $\cdot$ A. Buck $\cdot$

M. Schwaiger

Department of Nuclear Medicine,

Technische Universität München,

Ismaningerstrasse 22,

81675 Munich, Germany

e-mail: mpiert@umich.edu

M. Burian · H. J. Stein · J. R. Siewert

Department of Surgery, Technische Universität München,

Munich, Germany

\section{J. Nährig}

Institute of Pathology, Technische Universität München,

Munich, Germany

\section{Picchio}

Department of Nuclear Medicine, Scientific Institute San Raffaele,

IBFM-CNR,

Milan, Italy

Present address:

M. Piert

Department of Radiology, Division of Nuclear Medicine,

University of Michigan Health System, University Hospital,

B1G505C, 1500 E. Medical Center Drive,

Ann Arbor, MI 48109-0028, USA tion, phantom studies were performed to evaluate the spatial resolution and sensitivity of the probe.

Results All known targeted tumour sites were identified by the positron probe. T/B ratios were generally high, with a mean $\mathrm{T} / \mathrm{B}$ ratio of 6.6 , allowing easy identification of most tumour sites. In one case of a hepatic metastasis, the T/B ratio of 1.34 was below expectations, since the preoperative $\left[{ }^{18} \mathrm{~F}\right]$ FDG PET scan was positive. The probe was instrumental in the localisation of three additional tumour lesions (two lymph nodes, one anastomotic ring) that were not immediately apparent at surgery. Phantom studies revealed that $\left[{ }^{18} \mathrm{~F}\right] \mathrm{FDG}$-containing gel (simulating tumour tissue), having 10 times more $\left[{ }^{18} \mathrm{~F}\right] \mathrm{FDG}$ than surrounding "normal" background gel, was clearly detectable in quantities as low as $15 \mathrm{mg}$. As measured in two cases, the absorbed radiation doses ranged from 2.5 to $8.6 \mu \mathrm{Sv} / \mathrm{h}$ for the surgical team to $0.8 \mu \mathrm{Sv} / \mathrm{h}$ for the aesthetician.

Conclusion $\left[{ }^{18} \mathrm{~F}\right] \mathrm{FDG}$-accumulating tumour tissues can be localised with positron probes intraoperatively with a low radiation burden to the patient and medical personnel. The methodology holds promise for further clinical testing.

Keywords Positron probe $\cdot{ }^{18}$ F-2-fluoro-2-deoxy-Dglucose . Tumour imaging Intraoperative tumour localisation

\section{Introduction}

Surgery can potentially cure locally metastasising solid tumours. Nevertheless, the presence of residual disease following conservative surgical treatment is a common cause of locally recurrent cancer and might also be the source of distant metastases. Reliable intraoperative identification of small cancer masses would permit the surgeon to 
selectively resect malignant tissue. Probe-guided surgery might allow the identification of tumour lesions that have remained undetected by preoperative imaging. Also, highly sensitive probe systems might be useful to differentiate tumour from scar after neoadjuvant cancer treatment and might allow the detection of small tumour masses at the level of resection margins.

Positron emission tomography (PET) with fluorine-18 labelled fluorodeoxyglucose $\left(\left[{ }^{18} \mathrm{~F}\right] \mathrm{FDG}\right)$ localises malignant tissue based on the up-regulation of glucose transporters and subsequent phosphorylation by hexokinase. Although the specificity of $\left[{ }^{18} \mathrm{~F}\right] \mathrm{FDG}$ for malignant tissue is limited, whole-body $\left[{ }^{18} \mathrm{~F}\right] \mathrm{FDG}$ PET imaging has been applied with tremendous success in a large number of malignancies for staging of initial and recurrent disease [1]. Additional oncological applications of PET are rapidly being developed and there is likely to be further expansion in applications with the availability of new positronemitting radiopharmaceuticals for clinical use [2-4]. Therefore, intraoperative hand-held probes that detect beta particles (positrons) emitted from the radioactive decay of fluorine-18 have recently been developed [5-10]. Selective detection of beta particles may be advantageous for the localisation of very small amounts (mg) of tumour tissue. Beta particles resulting from the decay of fluorine-18 have a mean range of only $0.5 \mathrm{~mm}$, and a maximum range of $2.4 \mathrm{~mm}$ in water before annihilation into two $511-\mathrm{keV}$ photons $[11,12]$. The short range of positrons in tissue necessitates primary localisation of suspected tumour tissue either visually by the surgeon or by using a high-energy gamma detector.

We tested a newly built commercially available beta and gamma probe system (SI PET-Probe System, Silicon Instruments, Berlin, Germany) that uses silicon detectors. The system is capable of simultaneous beta particle and high-energy photon detection using two separate probes.

\section{Materials and methods}

\section{Beta probe}

The detector unit of the intraoperative beta-probe system used in this study consists of a silicon detector incorporated into a single compact unit. The detector's design has been optimised for a high beta particle sensitivity and low absorption of detector-penetrating photons. The shaft of the probe has a diameter of $11 \mathrm{~mm}$ while the tip of the detector has an outer diameter of $16 \mathrm{~mm}$ with an active circular detector area of $20 \mathrm{~mm}^{2}$ at the tip of the probe. The front face of the tip is covered with an extremely thin layer of light-impenetrable foil to ensure insensitivity to light and electromagnetic shielding. Electrical connections from the detectors to the electronics boards located in the probe handle are routed through a stainless steel tube connecting the probe tip and handle. The probe weighs $65 \mathrm{~g}$ and is easily handled by the surgeon (Fig. 1).

\section{High-energy gamma probe}

The high-energy gamma probe detector unit is a shielded silicon detector with a $\mathrm{CsI}(\mathrm{Tl})$ scintillation crystal gamma probe weighting $160 \mathrm{~g}$. As recommended by the manufacturer, the energy threshold for $511-\mathrm{keV}$ photon detection was set to $460 \mathrm{keV}$, electronically discarding photons with lower energy. The tip of the detector has an outer diameter of $11 \mathrm{~mm}$ with an active circular detector area of $16 \mathrm{~mm}^{2}$ at the tip of the probe. All measurements were done using a tungsten-shielded high-energy collimator provided with the system, which narrows the detector window to a diameter of $8 \mathrm{~mm}$ (Fig. 1).

For both probes, the variable-height pulses produced by the charge amplifiers were transmitted to the power- and pulse-processing module through $350 \mathrm{~cm}$ of shielded, multi-conductor cable. Sealed, watertight connectors were used to exclude the potential for electrical shock during use in surgery. Further technical specifications were not disclosed by the manufacturer.

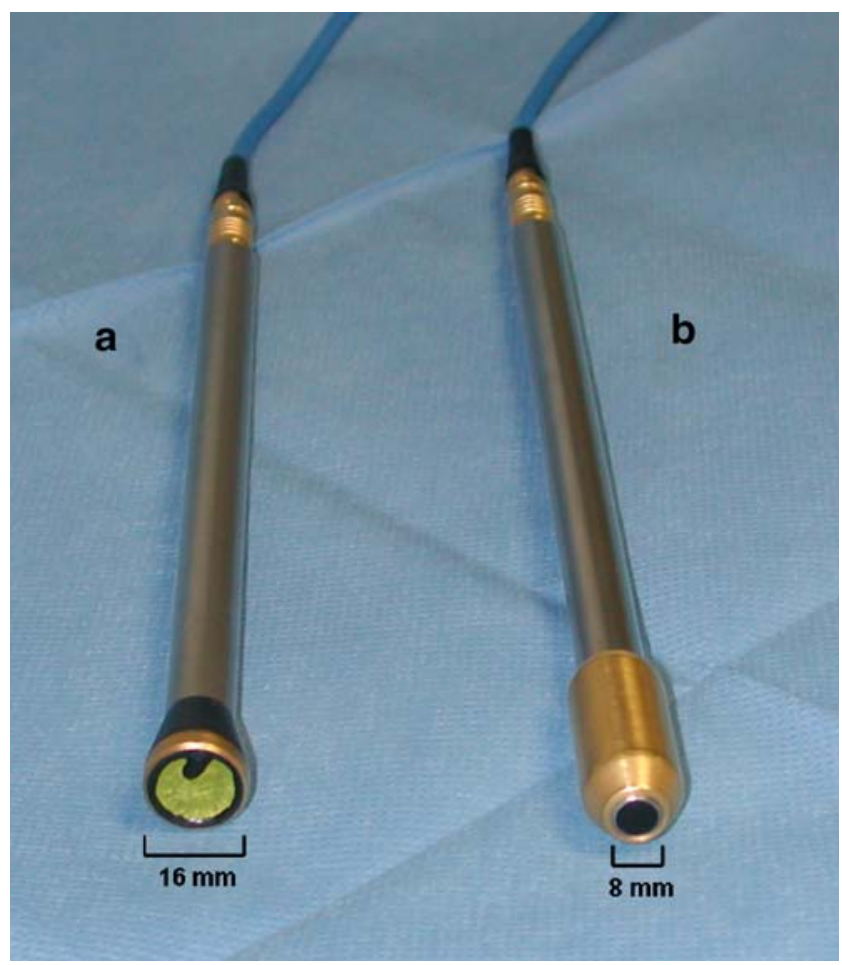

Fig. 1 Positron (a) and collimated high-energy gamma (b) probes manufactured by Silicon Instruments (Berlin, Germany) 
In vitro performance tests

Probe performance for the detection of beta particles and 511-keV gamma rays (photons) was tested using $\left[{ }^{18} \mathrm{~F}\right] \mathrm{FDG}$ solution.

\section{Sensitivity and linearity measurements}

The counting rates of the probe were measured in air as a function of source strength over $26.3 \mathrm{~h}$ at $1-\mathrm{mm}$ distance from a point source of $\left[{ }^{18} \mathrm{~F}\right] \mathrm{FDG}$ (dried $1-\mu \mathrm{l}$ drop of $\left[{ }^{18} \mathrm{~F}\right]$ FDG solution of less than $500 \mu \mathrm{m}$ diameter). The amount of radioactivity ranged from 409 to $0.06 \mathrm{kBq}$ over the length of the experiment. For annihilation photon detection testing, the point source was covered with a $1.0-\mathrm{mm}$-thick and 20-mm-wide piece of aluminium, sufficiently absorbing all beta particles emitted. Thus, the detector unit was bombarded with a pure flux of aluminium-penetrating 511$\mathrm{keV}$ annihilation photons. The beta and photon data were fitted as a function of radioactivity using linear regression analysis, obtaining the sensitivity for beta particles and annihilation photons.

\section{Resolution measurements}

Resolution measurements were performed with the positron probe. The tip of the probe was positioned $1.0 \mathrm{~mm}$ above a dried $185 \mathrm{kBq}\left[{ }^{18} \mathrm{~F}\right] \mathrm{FDG}$ point source. This point source was then moved in $1.0-\mathrm{mm}$ steps to both sides of the probe's tip, which was either covered with a $60( \pm 4) \mu \mathrm{m}$ thick latex foil (Microtek Probe Cover, Bremen, Germany) or uncovered. The full-width at half-maximum (FWHM) measurement was calculated from counts relative to $50 \%$ of maximum.

\section{Probe phantom measurements}

A simple phantom was used to simulate the detection of small amounts of superficial tumour tissue within background activity resulting from normal tissues. Briefly, a 12cm-diameter cylindrical plastic container was filled with $5 \mathrm{MBq}$ of $\left[{ }^{18} \mathrm{~F}\right] \mathrm{FDG}, 30 \mathrm{~g}$ of gelatine and $200 \mathrm{ml}$ of $\mathrm{H}_{2} \mathrm{O}$, stirred and refrigerated for $5 \mathrm{~h}$ until a semisolid, flat gel resulted, simulating normal background tissue. Various amounts of equally dense gel $(15,25,75,250$ or $1,000 \mathrm{mg}$ ), having a $\left[{ }^{18} \mathrm{~F}\right] \mathrm{FDG}$ concentration 10 times higher than background, were used to simulate tumour tissue. Cubic pieces of "tumour" gel were implanted into the "background" gel, retaining a flat surface. The latex foil-covered positron and gamma probe heads were advanced in millimetre steps within $10 \mathrm{~mm}$ of the "tumour" gel's centre and in 2-mm steps for the remainder of a 2-cmwide area around the "tumour" centre (Fig. 2). All

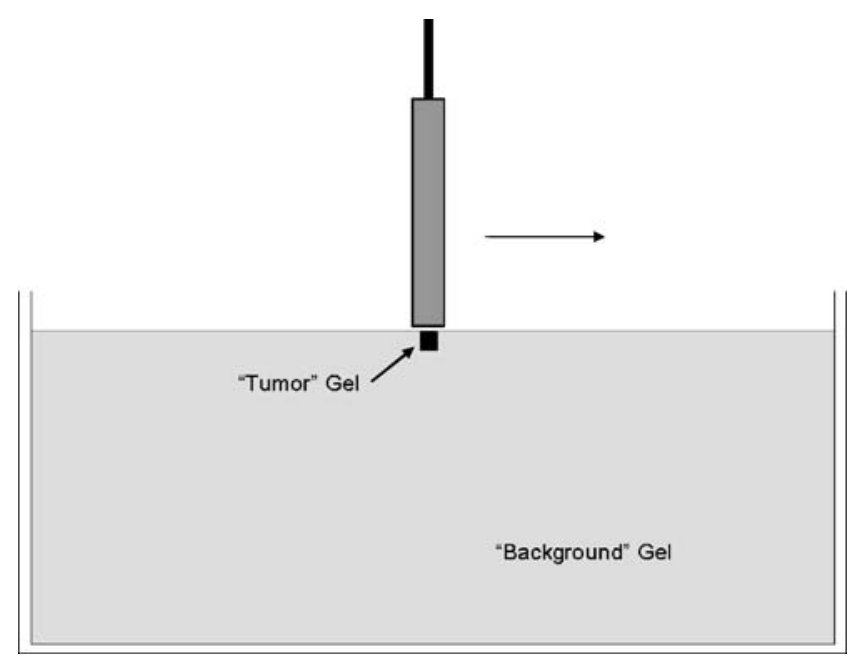

Fig. 2 Phantom studies using the positron and gamma detector probe were performed on a "tumour" gel at the surface of a large "background" gel. The probe's head was covered by $60-\mu \mathrm{m}$ thin latex foil as used during surgery (see Fig. 5 for results)

measurements were corrected for physical decay and performed in triplicate.

\section{PET phantom measurements}

A cylindrical phantom containing 9.41 of water was filled with $37 \mathrm{MBq}$ of $\left[{ }^{18} \mathrm{~F}\right] \mathrm{FDG}$ as background. Fifteen, 25, 75, 250 or $1,000 \mu \mathrm{l}$ of $\left[{ }^{18} \mathrm{~F}\right] \mathrm{FDG}$ solution containing 10 times more activity than background was filled into five appropriately sized spheres. A 10 -min static 3D acquisition was started on an ECAT EXACT HR+ PET scanner (Siemens/CTI, Knoxville, TN, USA). Image data were reconstructed using ordered subset expectation maximisation (OSEM, four iterations, 16 subsets). Attenuation correction was applied using a measured transmission scan. Image data were corrected for physical decay, random counts, dead time and scatter. Images were visually inspected for hot spots, and volumes of interest were defined in areas of increased activity to calculate the target to background ratio.

In vivo performance tests

Patients considered surgical candidates underwent informed consent under approved study by the Technical University of Munich institutional review board. A total of 17 patients with histologically proven malignancies (five adenocarcinomas of the distal oesophagus and gastrooesophageal junction, three gastric adenocarcinomas, one thyroid cancer, one breast cancer, two malignant melanomas, five colorectal cancers) were included. All patients underwent routine clinical staging including endoscopy and CT imaging as needed. Four of the five patients with 
adenocarcinomas of the distal oesophagus and gastrooesophageal junction received neoadjuvant chemotherapy. Based on an established protocol at our institution, $\left[{ }^{18} \mathrm{~F}\right]$ FDG PET was performed before and 2 weeks after the start of chemotherapy. According to that protocol, two patients were considered as non-responders at PET while the remaining two were classified as partial responders [13]. Therefore, all four patients underwent emergency surgery before completion of the chemotherapy protocol. The patient's history, PET imaging and probe measurement results are summarised in Table 1. Intraoperative probe readings were performed on average $3 \mathrm{~h}$ after tracer injection.

$\left[{ }^{18} \mathrm{~F}\right] \mathrm{FDG}$ PET or PET/CT scanning was performed in a fasted state with glucose levels less than $120 \mathrm{mg} / \mathrm{dl}$ within 2 weeks prior to surgery. Due to the fact that some patients required urgent treatment and PET or PET/CT was not immediately available, PET scanning could only be performed in 12 of the 17 patients. Emission images covering the area from the neck to the pelvis (seven or eight bed positions, 5-7 min duration each) were acquired starting 60 min after injection of 300-400 MBq using an ECAT EXACT HR+ PET scanner (followed by a measured transmission scan) or on a Biograph Sensation 16 PET/CT scanner using the $\mathrm{CT}$ data for attenuation correction (Siemens/CTI, Knoxville, TN, USA). Image data, corrected for dead time, random events, physical decay and scatter were reconstructed iteratively using attenuation-weighted OSEM algorithms (eight iterations, four subsets).

Standardised uptake values (SUVs) were calculated from an oval region of interest (ROI) measuring $1.5 \mathrm{~cm}^{2}$ which was placed in the transaxial slice having the maximum tumour uptake on PET or PET/CT images. A recent study comparing SUV measurements obtained with these two scanners showed insignificant differences of the SUV measurements, indicating that results are largely interchangeable if identical OSEM reconstruction parameters are chosen [14].

All patients underwent surgery including probe-directed localisation of $\left[{ }^{18} \mathrm{~F}\right] \mathrm{FDG}$ after intravenous injection of 36 $110 \mathrm{MBq}\left[{ }^{18} \mathrm{~F}\right] \mathrm{FDG}$ prior to anaesthesia. The amount of tracer injected was determined according to the projected time span between the start of the procedure and the time of intraoperative measurements. Intravenous fluids were void of carbohydrates. At surgery, the probe was covered with a sterile latex foil (same type as was used for in vitro testing). The operational field was scanned and absolute counts per second (cps) were determined at the known tumour sites and normal tissue of the same organ (at least $5 \mathrm{~cm}$ away from macroscopically visible tumour tissue). Counts were recorded in real time and visualised on the system's monitor as cps. In addition, the system provided auditory signals

Table 1 Patient characteristics, positron probe and PET measurements

\begin{tabular}{|c|c|c|c|c|c|c|c|}
\hline No. & $\begin{array}{l}\text { Age } \\
(\mathrm{yr})\end{array}$ & $\begin{array}{l}\text { Body weight } \\
(\mathrm{kg})\end{array}$ & $\begin{array}{l}\text { Inj. activity } \\
(\mathrm{MBq})\end{array}$ & $\begin{array}{l}\mathrm{TD} \\
(\mathrm{min})\end{array}$ & $\begin{array}{l}\mathrm{T} / \mathrm{B} \\
\text { ratio }\end{array}$ & $\mathrm{SUV}_{\text {mean }}$ & Histology \\
\hline 1 & 83 & 69 & 93.6 & 153 & 9.0 & 6.8 & Metastasis of papillary thyroid cancer \\
\hline 2 & 40 & 88 & 47.0 & 80 & 3.5 & 5.3 & Gastric adenocarcinoma \\
\hline 3 & 69 & 86 & 65.5 & 133 & 4.9 & 8.3 & $\begin{array}{l}\text { Adenocarcinoma of the gastro-oesophageal } \\
\text { junction }\end{array}$ \\
\hline 4 & 56 & 70 & 41.4 & 365 & 3.4 & 6.6 & Recurrent rectal cancer \\
\hline 5 & 43 & 86 & 110.6 & 300 & 3.2 & - & Adenocarcinoma of the colon \\
\hline 6 & 75 & 70 & 44.0 & 140 & 5.2 & - & Adenocarcinoma of the colon \\
\hline 7 & 63 & 89 & 73.6 & 199 & 1.3 & 7.2 & Liver metastasis of colon cancer \\
\hline 8 & 76 & 66 & 48.5 & 149 & 4.1 & 4.1 & Gastric adenocarcinoma \\
\hline 9 & 48 & 72 & 40.7 & 189 & 2.6 & 4.9 & Barret's cancer \\
\hline 10 & 60 & 86 & 41.1 & 105 & 5.6 & 2.8 & Ductal breast cancer \\
\hline 11 & 57 & 58 & 36.6 & 165 & 10.4 & 2.3 & Adenocarcinoma of the distal oesophagus \\
\hline 12 & 67 & 95 & 52.9 & 149 & 17.2 & - & Adenocarcinoma of the distal oesophagus \\
\hline 13 & 59 & 73 & 36.3 & 250 & 3.3 & 12.8 & Malignant melanoma \\
\hline 14 & 73 & 86 & 37.7 & 19 & 12.3 & 5.0 & Malignant melanoma \\
\hline 15 & 65 & 84 & 36.6 & 171 & 8.4 & 3.8 & Adenocarcinoma of the colon \\
\hline 16 & 71 & 62 & 37.7 & 255 & 16.3 & - & Gastric adenocarcinoma \\
\hline 17 & 53 & 85 & 40.7 & 239 & 11.7 & - & $\begin{array}{l}\text { Adenocarcinoma of the gastro-oesophageal } \\
\text { junction }\end{array}$ \\
\hline Mean & 62.3 & 77.9 & 52.0 & 184.3 & 6.6 & 5.8 & \\
\hline SD & 12.0 & 11.0 & 21.7 & 72.2 & 4.8 & 2.8 & \\
\hline
\end{tabular}

$T D$ time difference between injection and probe measurements, $T / B$ tumour/background ratio, $S U V_{\text {mean }}$ mean standardised uptake value in tumour obtained from $\left[{ }^{18} \mathrm{~F}\right] \mathrm{FDG}$ PET or PET/CT imaging 


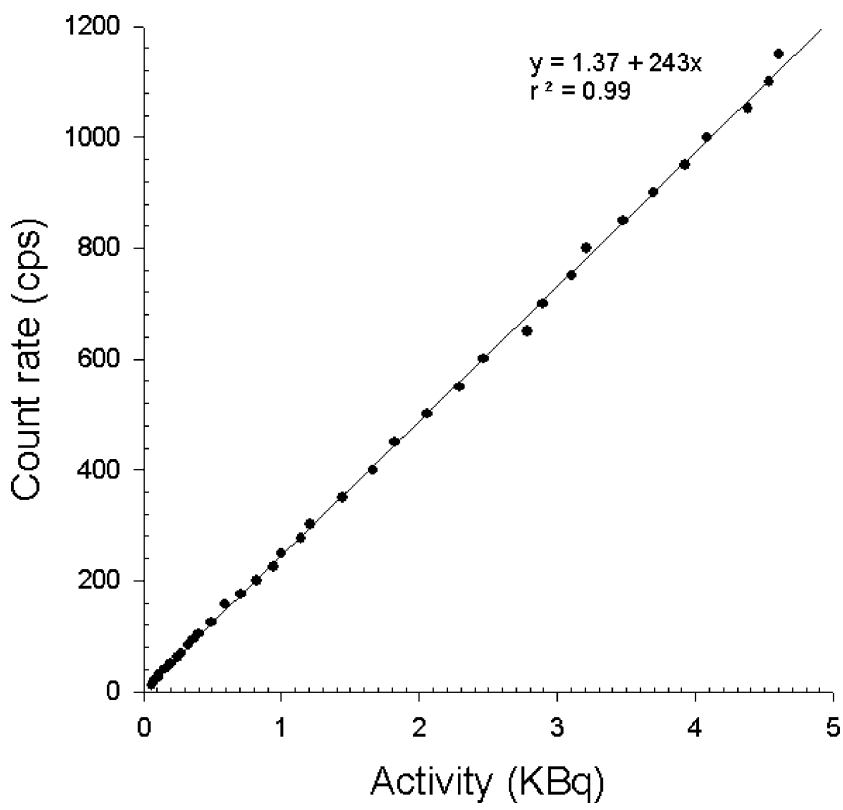

Fig. 3 Sensitivity testing of the positron probe revealed approximately 250 counts per second (cps) recovered from a point source of $1 \mathrm{kBq}$ of $\left[{ }^{18} \mathrm{~F}\right] \mathrm{FDG}$

indicating the level of count reading. For further data analysis, measurements were obtained in triplicate using a gate time of $10 \mathrm{~s}$. The time interval between $\left[{ }^{18} \mathrm{~F}\right] \mathrm{FDG}$ injection and measurement was recorded. The operative procedure was otherwise conducted as planned. The lesionto-background ratio was calculated using the normal tissue of the respective tumour-involved organ as background. In two cases, a suspiciously enlarged lymph node was identified by the surgeon. Probe readings were performed from the suspicious as well as the non-involved lymph nodes for comparison. In one additional case, anastomotic rings were evaluated using the probe. For comparison, counts were obtained from normal anastomotic rings (aboral anastomosis).

\section{Radiation dose measurements}

Absorbed doses resulting from the decay of $\left[{ }^{18} \mathrm{~F}\right] \mathrm{FDG}$ in the operation room were measured with a calibrated digital dosimeter (Isotrak DoseGUARD S10, Braunschweig, Germany) for the most affected surgeon, his first assistant and the aesthetician during two procedures. The radiation detectors were worn beneath clothing at the level of the anterior chest. All patients received a Foley catheter to reduce urinary activity during the procedure.

\section{Statistics}

Linear fitting was performed with Sigma Plot 8.0 (SPSS Science). Data are presented as mean \pm standard deviation of measurements.

\section{Results}

In vitro measurements

The sensitivity of the beta probe for fluorine-18 positron detection was measured as $0.25 \mathrm{cps}$ per $\mathrm{Bq}$ ( $250 \mathrm{cps}$ per $\mathrm{kBq}$ ) at a distance of $1 \mathrm{~mm}$ from a point source without cover (Fig. 3). Positron sensitivity decreased on average by $33 \%$ using the intraoperative $60-\mu \mathrm{m}$-thick latex cover. The probe's sensitivity to penetrating $511-\mathrm{keV}$ photons was tested by shielding the probe's tip with a 1-mm-thick lead cover and found to be negligible (less than $1.5 \%$ of total counts at any count rate up to $1,000 \mathrm{cps}$ ) (Fig. 4). At a distance of $10 \mathrm{~mm}$ to the point source, the sensitivity for positrons decreased by a factor of 5 , while the ratio between true positron detection and background detection from penetrating photons increased slightly to $3.5 \%$ of total counts.

Linearity was maintained in the clinically important range (20-1,000 cps) and above to a count rate of approximately 10,000 cps (equivalent to approx. $50 \mathrm{kBq}$ at point source). At higher activities $(>50 \mathrm{kBq})$, linearity was increasingly lost owing to dead time (data not shown).

The sensitivity of the high-energy photon probe for fluorine- 18 was measured to be $0.066 \mathrm{cps}$ per kilobecquerel at a distance of $1 \mathrm{~mm}$ from a point source without any cover (data not shown). Linearity was maintained throughout the investigated range of activity up to $500 \mathrm{kBq}$. However, the sensitivity of this high-energy photon probe

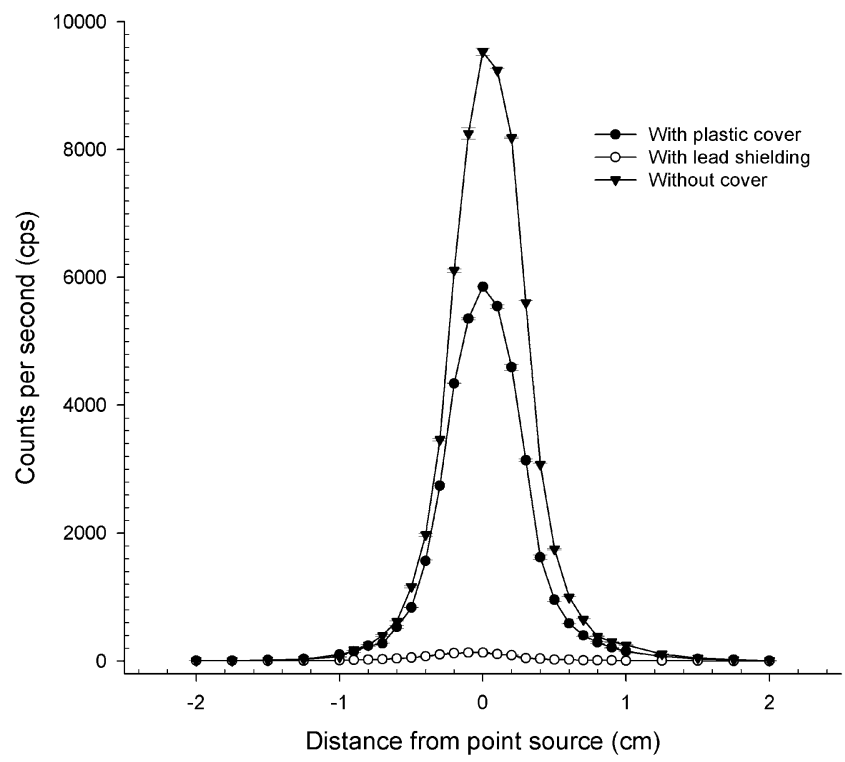

Fig. 4 In vitro resolution testing of the positron probe with a fluorine18 point source at $1-\mathrm{mm}$ distance from the point source. Use of a 60 $\mu \mathrm{m}$ thin plastic cover (black circles) decreased sensitivity by roughly one-third compared with uncovered measurements (black triangles). Lead shielding (white circles) demonstrates the insensitivity of the positron probe to penetrating $511-\mathrm{keV}$ photons 
was considered too low to allow further in vivo testing. Consequently, intraoperative measurements were only performed with the beta probe.

At $1 \mathrm{~mm}$ distance from point source, the FWHM of the positron probe was determined to be approximately $5 \mathrm{~mm}$ (Fig. 4). At 10-mm distance from source, the resolution decreased slightly to an FWHM of approximately $8 \mathrm{~mm}$.

\section{Phantom measurements}

Phantom measurements were performed with the beta and high-energy photon probes covered with latex foil. Without the presence of background activity, the net decay-corrected beta counting rate for "tumour" gel was indeed nearly 10 times higher than that for "background" gel (9.6:1 ratio). Nevertheless, this ratio was not recovered during phantom measurements. The counting ratio between "tumour" and "background" gel decreased with the reduction in size of the "tumour" gel (3.6:1 for $1 \mathrm{~g}, 2.8: 1$ for $250 \mathrm{mg}, 2.5: 1$ for $75 \mathrm{mg}, 2.0: 1$ for $25 \mathrm{mg}$ and 1.5:1 for the $15 \mathrm{mg}$ "tumour" gel). Figure 5 displays these ratios, when the count rate at the centre of the tumour (normalised to $100 \%$ ) is divided by the mean activity measured between 1.6 and $2 \mathrm{~cm}$ from the centre. Even when the probe's head was covered with the latex foil, $15 \mathrm{mg}$ of "tumour" gel was clearly localised by the positron probe given the selected "tumour"-to-"background" ratio of 10:1.

High-energy photon measurements revealed a much lower T/B ratio of 1.4:1 for the $1 \mathrm{~g}$ "tumour" gel, and 1.3:1 for the $250 \mathrm{mg}$ "tumour" gel, while smaller "tumour" gels

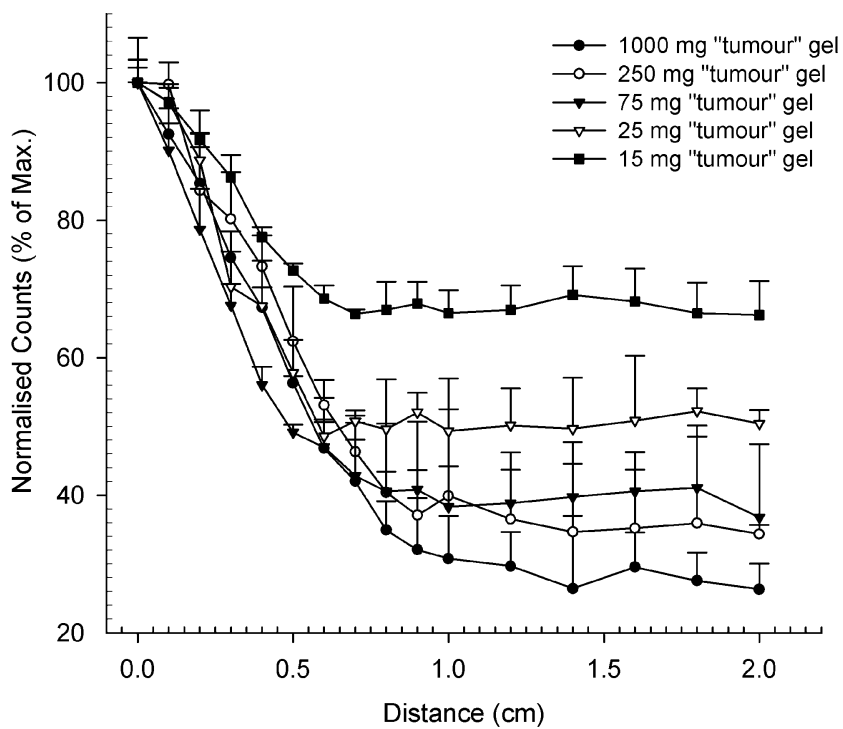

Fig. 5 Simulation of "tumour" tissue detection within background activity. Detection of variously sized (between 15 and 1,000 mg) highactivity gel masses (simulating tumour tissue) within background activity having 10 times less activity compared with the target using nonpressured direct gel contact. As during surgery, measurements were obtained using a $60-\mu \mathrm{m}$ thin latex foil cover remained undetected. For this reason, in conjunction with its low sensitivity, the high-energy probe was not considered for further intraoperative testing.

\section{PET phantom measurements}

On the 10-min static acquisition image of the phantom, only the 250 - and $1,000-\mu 1$ spheres were visually identified, while smaller spheres containing 15,25 and $75 \mu \mathrm{l}$ of $\left[{ }^{18} \mathrm{~F}\right]$ FDG solution remained undetected. Target to background ratios were 2.7:1 for the 1,000-mg sphere and 2.1:1 for the $250-\mathrm{mg}$ sphere, indicating significant partial volume effects.

In vivo measurements

Intraoperative tests have verified the feasibility of positron detection using this device by detecting all primarily targeted tumour sites. Known tumour tissue was clearly detected by the beta probe in 16 of 17 cases. The T/B ratios of primary targeted tumour lesions varied from 1.34:1 to 17.2:1, resulting in a T/B ratio of $6.6 \pm 4.8($ mean $\pm \mathrm{SD})$ for the entire patient population. However, one $\left[{ }^{18} \mathrm{~F}\right] \mathrm{FDG}$ PETpositive liver metastasis of a colorectal cancer having a tumour-to-background ratio of 1.34 was difficult to localise during the procedure based on positron probe readings alone.

Besides these primary targeted tumour sites, several additional $\left[{ }^{18} \mathrm{~F}\right]$ FDG-positive foci were identified using the positron probe during the procedures. In one case, a resection margin at the gastro-oesophageal anastomosis (stapler anastomosis) was found to have mildly increased counts at a small portion of the circumference (T/B ratio 1.3:1). Histology revealed the presence of focal tumour tissue at the level of the resection margin. Also, an adenocarcinoma of the distal oesophagus barely visualised on preoperative $\left[{ }^{18} \mathrm{~F}\right] \mathrm{FDG}$ PET imaging was clearly identified by intraoperative probe measurements (Table 1, case 11). Intraoperative positron probe scanning of the operation field revealed two additional $\left[{ }^{18} \mathrm{~F}\right] \mathrm{FDG}$-positive foci (T/B ratio 1.8 and 3.4) in two patients identified by the surgeon as lymph nodes close to the tumour site (Table 1, cases 2 and 6). Histological analysis of these lesions verified the presence of lymph node metastases.

Correlation of imaging and intraoperative beta probe findings

SUV measurements from PET and PET/CT imaging and correlative intraoperative probe measurements in relation to the injected dose and time span between tracer injection and probe readings are summarised in Table 1 . Pre-operative PET images of patient 11 with a primary distal oesophageal cancer are shown in Fig. 6, displaying only mildly 
increased metabolic activity (mean SUV 2.3) at the tumour site. Intraoperative measurements obtained from the tumour surface and normal oesophagus as background revealed a $\mathrm{T} / \mathrm{B}$ ratio of 10.4 , indicating that $\mathrm{PET}$ and probe readings do not necessarily correlate. Indeed, regression analysis performed from patients for whom PET imaging was available $(n=12)$ revealed no significant correlation between the T/B ratio obtained from probe readings and SUV measurements $\left(r^{2}=0.15\right.$; n.s. $)$.

\section{Radiation dose measurements}

Radiation dose measurements were performed during two procedures (patients 2 and 4). The length of the procedures ranged between 2 and $6.25 \mathrm{~h}$. Absorbed radiation doses ranged from 2.5 to $8.6 \mu \mathrm{Sv} / \mathrm{h}$ for the most affected surgeon to $0.8 \mu \mathrm{Sv} / \mathrm{h}$ for the aesthetician.

\section{Discussion}

Many solid cancers that metastasise to regional lymph nodes or, in the case of gastrointestinal cancers, even to distant organs can potentially be cured by surgical resection [15]. Minimally invasive techniques have been adapted to surgical oncology, decreasing morbidity and addressing the quest for optimal function after tumour resection [16-19]. While current preoperative imaging modalities (ultrasound,

Fig. 6 Transaxial (a) and coronal (b) $\left[{ }^{18} \mathrm{~F}\right]$ FDG PET images of patient 11 , displaying a rather low metabolic activity in a distal oesophageal cancer (mean SUV 2.3). Intraoperative probe readings verified a high tumour-tobackground ratio of 10.4 at the surface of the oesophageal cancer. The presence of viable tumour tissue at this location was verified at histology
X-ray, CT, MRI, PET, etc.) are used with the intention of determining a patient's eligibility for surgery, their overall performance in identifying all potential metastases remains suboptimal. Nevertheless, PET has evolved as a powerful tool for the detection of a large number of cancers. A variety of radiopharmaceuticals have been developed for PET imaging of cancer, including those related to increased amino acid transport, DNA synthesis, somatostatin receptors and tumour hypoxia $[2,4,20-22]$, but only $\left[{ }^{18} \mathrm{~F}\right] \mathrm{FDG}$ has as yet been approved for human use by the FDA. The physical properties of $\left[{ }^{18} \mathrm{~F}\right] \mathrm{FDG}$ make this molecule especially useful for intraoperative tumour imaging. The positron (positively charged electron) emitted from the proton-rich/neutron-deficient isotope travels only a rather short (mean range in water $=0.5 \mathrm{~mm}$ [11]) distance in tissue before interaction with a negatively charged electron and subsequent annihilation, resulting in the emission of two 511-keV photons, which are used for coincidence imaging (PET) and for identification with photon-sensitive highenergy (gamma) probes, giving an approximation to the location of maximum positron emission [23]. $\left.{ }^{18} \mathrm{~F}\right] \mathrm{FDG}$ accumulation in tumour tissue is based on high cellular uptake, glycolytic rate and subsequent trapping, yielding excellent tumour-to-background ratios frequently greater than 10:1 [24].

Most current intraoperative probes are designed for the detection of relatively low-energy photons emitted from isotopes such as technetium- $99 \mathrm{~m}(140 \mathrm{keV})$ or indium-111
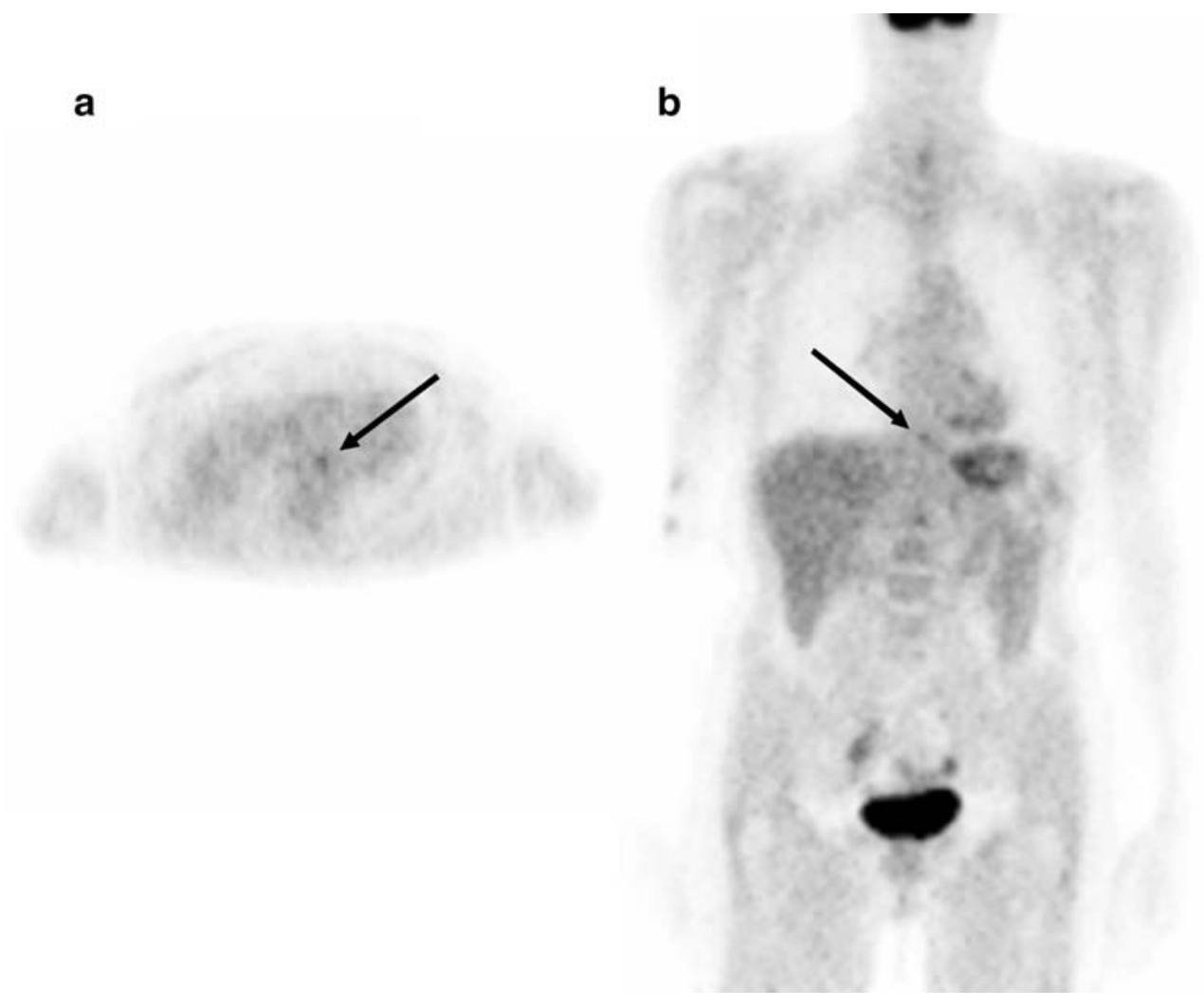
$(170 \mathrm{keV} / 245 \mathrm{keV})$ and are, therefore, not well suited for high-energy gamma or positron detection [9]. Existing gamma probes have been used for the detection of positron emitters, but their performance remains well below the expected performance of dedicated positron probes [7]. Recently, two studies reported on the intraoperative use of gamma probes specifically designed for the detection of high-energy photons resulting from the decay of $\left[{ }^{18} \mathrm{~F}\right] \mathrm{FDG}$. Using a prototype probe in head and neck cancer patients, Meller et al. found tumour-to-background ratios between 1.2 and 4.9 and a high sensitivity (95\%) for the detection of metastatic lymph nodes [25]. Gulec et al. investigated 32 patients with various malignancies using a different highenergy gamma probe [26]. All PET-positive lesions were detected intraoperatively with tumour-to-background ratios below 3. Injected radiotracer doses were in the range of 250-370 MBq $\left[{ }^{18} \mathrm{~F}\right] \mathrm{FDG}$ in these two studies, and thus significantly higher than in this study.

A dedicated positron probe composed of a plastic scintillator and a bismuth germanate (BGO) crystal was developed by Yamamoto et al. [27]. The FWHM of this probe was determined as $11 \mathrm{~mm}$ at $5-\mathrm{mm}$ distance from source, while the sensitivity for positrons was rather low ( $2.6 \mathrm{cps} / \mathrm{kBq}$ measured $5 \mathrm{~mm}$ from the detector surface). Raylman and Wahl developed a detector unit consisting of a stack of two ion-implanted silicon detectors [28]. The sensitivity for the positron detection was found to be approximately $200 \mathrm{cps} / \mathrm{kBq}$, while the resolution (in FWHM) was not reported [29]. Zervos et al. investigated a "beta"-sensitive probe manufactured by Crystal (Berlin, Germany) in five patients. While technical specifications, sensitivity and resolution characteristics of this probe were not specifically stated, the measured tumour to background ratios after $\left[{ }^{18} \mathrm{~F}\right] \mathrm{FDG}$ administration were generally low (mean 1.6:1) and not different from those measured with a gamma probe (Neoprobe Corporation, Dublin, $\mathrm{OH}$ ) using high energy window settings [30]. Daghighian et al. built a now commercially available dedicated positron probe (IntraMedical, Los Angeles, CA, USA) that uses two detectors, one that predominantly detects positrons and another that detects only gamma rays, allowing the subtraction of background gamma activity from "true" positron counts. They reported a sensitivity of $108 \mathrm{cps} / \mathrm{kBq}$ and a resolution of $10 \mathrm{~mm}$ (FWHM) for a source of $5-\mathrm{mm}$ width [31]. Essner et al. reported successful detection of remaining tumour tissue in a single case of a melanoma metastasis in the neck [8], while Frac et al. investigated 5 patients with recurrent melanoma yielding a sensitivity of $89 \%$ and a specificity of $100 \%$ using this device [32].

Physical testing results obtained with the positron probe used in this study compare favourably with previously tested detectors. At 1-mm distance from a point source of $\left[{ }^{18} \mathrm{~F}\right] \mathrm{FDG}$, the sensitivity for positrons was found to be roughly $250 \mathrm{cps} / \mathrm{kBq}$, while the FWHM was calculated as $5 \mathrm{~mm}$ using a point source. At $10-\mathrm{mm}$ distance, sensitivity decreased fivefold and the FWHM was found to be $8 \mathrm{~mm}$, indicating that performance is best when direct contact of the probe with the tissue is established. Covering the probe with a standard intraoperative ultrasound latex foil (as done in this study) decreases the sensitivity significantly. The penetration of positrons through the plastic foil is strongly dependent on the thickness of the material and may therefore vary between available products. Since the positron probe used in this study cannot be gas sterilised, this necessitates a moderate increase in the administered dose for intraoperative use.

Phantom studies performed with the positron probe covered with a latex foil revealed that $\mathrm{T} / \mathrm{B}$ ratios suitable for intraoperative use were achieved with simulated tumour tissue as small as $15 \mathrm{mg}$. Data published by Raylman et al. [33] as well as our own phantom study indicate that tumour lesions have to be at least one order of magnitude larger to be detected under clinical scanning conditions using highresolution PET scanners. If gas sterilisation were possible, probe measurements without latex foil would increase the sensitivity by approximately one-third, suggesting that even smaller lesions would become detectable. Thus, under optimal intraoperative measurement conditions only a few milligrams of superficial tumour tissue would be necessary for detection.

To our knowledge, this study is the first to utilise a dedicated positron probe (beta detection only) for intraoperative detection of tumour tissue in a series of patients. All primarily targeted tumours had higher counts than adjacent normal tissue, and all but one had a T/B ratio greater than $1.5: 1$, allowing easy in vivo detection of the primarily targeted tumour tissue. The suboptimal T/B ratio in a single case of a liver metastasis despite preoperative visualisation by $\left[{ }^{18} \mathrm{~F}\right]$ FDG PET scanning may be due to individual changes of the hepatic glucose metabolism during surgery. It is well known that general anaesthesia and intraoperative stress can have profound effects on liver metabolism and ultimately may alter hepatic $\left[{ }^{18} \mathrm{~F}\right] \mathrm{FDG}$ uptake as well $[34,35]$. Also, since positrons resulting from the decay of fluorine-18 travel only up to a maximum range of $2.4 \mathrm{~mm}$ in tissue, even a thin layer of non-malignant tissue above the lesion may render it impossible to detect the malignancy, because the majority of positrons will originate from the most superficial tissues and not from the cancer beneath it. Subsequent studies are needed to investigate this effect by measuring the thickness of normal tissue above tumour at the site of measurement.

Intraoperative testing revealed that a ratio between target tissue and background was most suitable for the detection of tumour tissue. One can identify the level of radioactivity at the tissue surface based on the measured counts in real time by moving the probe's head in the surgical field from tumour to adjacent normal background tissue of the same 
organ and back. But since the system did not provide the ability to set a certain threshold as background activity, a calculated T/B ratio was used instead of raw counts for further analysis.

The spatial resolution of the probe decreases with the size of the detector head, while sensitivity increases with detector size. However, the spatial resolution is almost irrelevant for the detection of tumour tissue that has at least the size of the detector head. In such cases, a high sensitivity is beneficial since it allows minimisation of the radiation burden to the patient and personnel. However, spatial resolution would become relevant if very small tumour masses (a few milligrams) not easily identifiable by the human eye were to be targeted. Then, a time-consuming scan of the surgical bed would be needed. Clearly, an imaging device based on positron detection that could be placed into the surgical field would be highly desirable.

The goal of this study was to examine the utility of the probe for identifying known malignancies and not to attempt to find tumour lesions not visualised by preoperative imaging. Nevertheless, during surgery two $\left[{ }^{18} \mathrm{~F}\right]$ FDG-positive foci were identified as being enlarged lymph nodes and later found to be metastatic lymph nodes at histology. In one case, anastomotic rings were investigated and one ring was found to be marginally positive based on probe measurements and later verified to contain tumour tissue at histology.

Intraoperative probe performance is dependent on two major factors, the metabolism of the administered radiopharmaceutical and the technical performance of the probe. Physiological parameters that influence the ability to detect tumour tissue in situ will include the time interval between tracer injection and scanning, the uptake of the tracer in tumour tissue compared with its surrounding normal tissue, and the clearance of the radiopharmaceutical from blood. In our experience, a minimum T/B ratio of 1.5 is needed for the operating surgeon to comfortably identify a significant difference between tumour and normal surrounding tissues. Since the uptake ratio between tumour and normal tissue generally increases with time, it seems advantageous to inject the radiopharmaceutical prior to the start of the procedure and to adjust the administered dose accordingly. Also, $\left[{ }^{18} \mathrm{~F}\right] \mathrm{FDG}$ administration before the start of the procedure should be preferred because the intraoperative glucose metabolism may be influenced in unpredictable ways by the surgical trauma as well as the anaesthetics used.

However, injection of the radiopharmaceutical prior to the start of surgery requires the use of a probe system that provides optimal sensitivity for positrons, because otherwise the administered radioactivity may result in a significant cumulative radiation dose for the surgical team, if frequent procedures are performed. The probe system used in this study showed the highest sensitivity of all positron probes published so far. As a result, the radiation burden to the patient and the surgical team can be substantially reduced compared with that associated with the use of high-energy gamma probes, while still maintaining a favourable high target-to-background ratio [25, 26]. Injected doses of $\left[{ }^{18} \mathrm{~F}\right] \mathrm{FDG}$ were rather low (range 36$110 \mathrm{MBq}$ ). Dose selections were made according to the anticipated time difference between injection and presumed intraoperative probe measurements, which can be troublesome in the event of unexpected procedural delays. In this study, intraoperative measurements of absorbed doses performed in a rather limited number of procedures were found to be low and ranged from 3 to $9 \mu \mathrm{Sv} / \mathrm{h}$ for the most affected surgeons to $0.8 \mu \mathrm{Sv} / \mathrm{h}$ for the aesthetician. Besides being dependent on the injected dose of $\left[{ }^{18} \mathrm{~F}\right] \mathrm{FDG}$, absorbed doses to personnel were essentially influenced by the distance from the patient's body and the duration of exposure. However, these preliminary data suggest that even frequent repetition of positron-guided surgical procedures would not exceed established dose limits for the operating room personnel as long as high-sensitivity probes are used.

Nevertheless, the in vivo testing of the positron probe revealed technical limitations that need to be addressed by the manufacturer. First, the relatively large diameter of the tip of the positron probe currently prevents its use for endoscopic procedures. However, a significantly smaller detector design would result in a loss of sensitivity, potentially limiting its in vivo usefulness. Also, the manufacturer was unable to resolve a technical issue in that intense pressure to the detector surface resulted in false readings. While this technical flaw could easily be prevented during procedures by using the probe with non-pressured direct tissue contact, this defect still limits its intraoperative applications. Most importantly, the sensitivity of the highenergy gamma probe provided with the system was much too low to be clinically applicable with the administered doses of $\left[{ }^{18} \mathrm{~F}\right]$ FDG. Still, the application of a high-energy gamma probe would be beneficial if the tumour tissue cannot be easily exposed during surgery or the tumour surface is covered by normal non-malignant tissues.

\section{Conclusion}

The study demonstrates the feasibility of localising $\left[{ }^{18} \mathrm{~F}\right]$ FDG-avid tumour tissue using a positron probe during surgery. The high sensitivity of the positron probe allowed procedures to be performed with a low radiation burden to the patient and personnel, while the high-energy gamma probe was not clinically useful owing to its low sensitivity. Based on phantom studies, the amount of tumour tissue needed for localisation is at least one order of magnitude smaller compared with whole-body $\left[{ }^{18}\right.$ F]FDG PET and the 
detection of very small $\left[{ }^{18} \mathrm{~F}\right] \mathrm{FDG}$ deposits (a few milligrams) is possible. In this study we have shown that positron probes are able to identify primary tumours, malignant lymph nodes and tumour infiltrates in anastomotic rings based on their $\left[{ }^{18} \mathrm{~F}\right] \mathrm{FDG}$ uptake during surgery. However, intraoperative metabolic changes may severely influence $\left[{ }^{18} \mathrm{~F}\right] \mathrm{FDG}$ uptake and may decrease the achievable tumour-to-background contrast (as demonstrated for a liver metastasis), indicating that close intraoperative monitoring of the glucose metabolism and proper timing of measurements is advisable.

As suggested by results from our phantom studies, the use of high-sensitivity positron probes may allow for the detection of remaining tumour tissue in resection beds. However, the detection of such small tumour lesions would necessitate a thorough and time-consuming exploration of the operative site, which in many cases may not be feasible. Given the short range of positrons in tissue, tumour sites that are covered with benign tissues may potentially remain unnoticed by positron detection alone. Combined positron/ high-energy gamma detectors with reasonably high sensitivity may be the best choice to overcome this limitation.

Acknowledgements None of the authors have any commercial interest or affiliation with any of the products discussed in the manuscript. The study was sponsored by the Clinic of Nuclear Medicine and Surgery at the Klinikum R. d. Isar of the Technical University of Munich, Germany.

\section{References}

1. Gambhir SS, Czernin J, Schwimmer J, Silverman DH, Coleman RE, Phelps ME. A tabulated summary of the FDG PET literature. J Nucl Med 2001;42:1S-93S.

2. Piert M, Machulla H-J, Picchio M, Reischl G, Ziegler S, Kumar P, et al. Hypoxia-specific tumor imaging with ${ }^{18} \mathrm{~F}$-fluoroazomycin arabinoside. J Nucl Med 2005;46:106-13.

3. Beer AJ, Haubner R, Goebel M, Luderschmidt S, Spilker ME, Wester HJ, et al. Biodistribution and pharmacokinetics of the \{alpha\} $\mathrm{v}$ \{beta\}3-selective tracer ${ }^{18} \mathrm{~F}$-galacto-RGD in cancer patients. J Nucl Med 2005;46:1333-41.

4. Picchio M, Treiber U, Beer AJ, Metz S, Bossner P, van Randenborgh $\mathrm{H}$, et al. Value of ${ }^{11} \mathrm{C}$-choline PET and contrastenhanced CT for staging of bladder cancer: correlation with histopathologic findings. J Nucl Med 2006;47:938-44.

5. Yamamoto S, Matsumoto K, Senda M. Optimum threshold setting for a positron-sensitive probe with background rejection capability. Ann Nucl Med 2004;18:251-6.

6. Raylman RR, Fisher SJ, Brown RS, Ethier SP, Wahl RL. Fluorine18-fluorodeoxyglucose-guided breast cancer surgery with a positron-sensitive probe: validation in preclinical studies. J Nucl Med 1995;36:1869-74.

7. Essner R, Hsueh EC, Haigh PI, Glass EC, Huynh Y, Daghighian F. Application of an $\left[{ }^{18} \mathrm{~F}\right]$ fluorodeoxyglucose-sensitive probe for the intraoperative detection of malignancy. J Surg Res 2001;96:120-6.

8. Essner R, Daghighian F, Giuliano AE. Advances in FDG PET probes in surgical oncology. Cancer J 2002;8:100-8.
9. Hoffman EJ, Tornai MP, Janecek M, Patt BE, Iwanczyk JS. Intraoperative probes and imaging probes. Eur J Nucl Med 1999;26:913-5.

10. Desai DC, Arnold M, Saha S, Hinkle G, Soble D, Fry J, et al. Correlative whole-body FDG-PET and intraoperative gamma detection of FDG distribution in colorectal cancer. Clin Positron Imaging 2000;3:189-96.

11. Bai B, Ruangma A, Laforest R, Tai Y-C, Leahy RM. Positron range modeling for statistical PET image reconstruction. IEEE $2003 ; 4: 2501-5$

12. Fowler JS, Wolf AP. Positron emitter-labeled compounds: priorities and problems. In: Phelps M, Mazziotta J, Schlebert H, editors. Positron emission tomography and autoradiography. Principles and applications for the brain and heart. New York: Raven Press; 1986. p. 391-450.

13. Weber WA, Ott K, Becker K, Dittler HJ, Helmberger H, Avril NE, et al. Prediction of response to preoperative chemotherapy in adenocarcinomas of the esophagogastric junction by metabolic imaging. J Clin Oncol 2001;19:3058-65.

14. Souvatzoglou M, Ziegler SI, Martinez MJ, Busch R, Dzewas G, Schwaiger M, et al. Standardised uptake values from PET/CT images: comparison with conventional attenuation-corrected PET. Eur J Nucl Med Mol Imaging 2006:[Epub ahead of print].

15. Mineo TC, Ambrogi V, Tonini G, Bollero P, Roselli M, Mineo D, et al. Longterm results after resection of simultaneous and sequential lung and liver metastases from colorectal carcinoma. J Am Coll Surg 2003;197:386-91.

16. Krag D. Minimal invasive staging for breast cancer: clinical experience with sentinel lymph node biopsy. Semin Oncol 2001;28:229-35.

17. Bojalian MO, Machado GR, Swensen R, Reeves ME. Radiofrequency ablation of liver metastasis from ovarian adenocarcinoma: case report and literature review. Gynecol Oncol 2004;93:557-60.

18. de Graaf EJ. Transanal endoscopic microsurgery. Scand J Gastroenterol Suppl 2003:34-9.

19. Greene FL. Laparoscopic management of colorectal cancer. CA Cancer J Clin 1999;49:221-8.

20. Wester HJ, Schottelius M, Scheidhauer K, Reubi JC, Wolf I, Schwaiger M. Comparison of radioiodinated TOC, TOCA and Mtr-TOCA: the effect of carbohydration on the pharmacokinetics. Eur J Nucl Med Mol Imaging 2002;29:28-38.

21. Weber WA, Wester HJ, Grosu AL, Herz M, Dzewas B, Feldmann $\mathrm{HJ}$, et al. $O-\left(2-\left[{ }^{18} \mathrm{~F}\right]\right.$ fluoroethyl)-L-tyrosine and L-[methyl- $\left.{ }^{11} \mathrm{C}\right]$ methionine uptake in brain tumours: initial results of a comparative study. Eur J Nucl Med 2000;427:542-9.

22. Buck AK, Schirrmeister H, Hetzel M, Von Der Heide M, Halter G, Glatting $\mathrm{G}$, et al. 3-deoxy-3-[ $\left[{ }^{18} \mathrm{~F}\right]$ fluorothymidine-positron emission tomography for noninvasive assessment of proliferation in pulmonary nodules. Cancer Res 2002;62:3331-4.

23. Levin CS, Hoffman EJ. Calculation of positron range and its effect on the fundamental limit of positron emission tomography system spatial resolution. Phys Med Biol 1999;44:781-99.

24. Wahl RL, Hutchins GD, Buchsbaum DJ, Liebert M, Grossman $\mathrm{HB}$, Fisher S. ${ }^{18} \mathrm{~F}$-2-deoxy-2-fluoro-D-glucose uptake into human tumor xenografts. Feasibility studies for cancer imaging with positron-emission tomography. Cancer 1991;67:1544-50.

25. Meller B, Sommer K, Gerl J, von Hof K, Surowiec A, Richter E, et al. High energy probe for detecting lymph node metastases with ${ }^{18} \mathrm{~F}-\mathrm{FDG}$ in patients with head and neck cancer. Nuklearmedizin 2006;45:153-9.

26. Gulec SA, Daghighian F, Essner R. PET-Probe: evaluation of technical performance and clinical utility of a handheld high-energy gamma probe in oncologic surgery. Ann Surg Oncol 2006 Jul 24; [Epub ahead of print].

27. Yamamoto S, Matsumoto K, Sakamoto S, Tarutani K, Minato K, Senda M. An intra-operative positron probe with background rejection capability for FDG-guided surgery. Ann Nucl Med 2005;19:23-8. 
28. Raylman RR, Wahl RL. Evaluation of ion-implanted-silicon detectors for use in intraoperative positron-sensitive probes. Med Phys 1996;23:1889-95.

29. Raylman RR. Performance of a dual, solid-state intraoperative probe system with ${ }^{18} \mathrm{~F},{ }^{99 \mathrm{~m}} \mathrm{Tc}$, and ${ }^{111} \mathrm{In}$. J Nucl Med 2001;42:352-60.

30. Zervos EE, Desai DC, DePalatis LR, Soble D, Martin EW. ${ }^{18} \mathrm{~F}-$ labeled fluorodeoxyglucose positron emission tomography-guided surgery for recurrent colorectal cancer: a feasibility study. J Surg Res 2001;97:9-13.

31. Daghighian F, Mazziotta JC, Hoffman EJ, Shenderov P, Eshaghian B, Siegel S, et al. Intraoperative beta probe: a device for detecting tissue labeled with positron or electron emitting isotopes during surgery. Med Phys 1994;21:153-7.
32. Franc BL, Mari C, Johnson D, Leong SP. The role of positron- and high-energy gamma photon probe in intraoperative localization of recurrent melanoma. Clin Nucl Med 2005;30:787-791.

33. Raylman RR, Kison PV, Wahl RL. Capabilities of two- and threedimensional FDG-PET for detecting small lesions and lymph nodes in the upper torso: a dynamic phantom study. Eur J Nucl Med 1999;26:39-45.

34. Obata R, Bito H, Ohmura M, Moriwaki G, Ikeuchi Y, Katoh T, et al. The effects of prolonged low-flow sevoflurane anesthesia on renal and hepatic function. Anesth Analg 2000;91:1262-8.

35. Molina PE, Ajmal M, Abumrad NN. Energy metabolism and fuel mobilization: from the perioperative period to recovery. Shock 1998;9:241-8. 